Pacific

Journal of

Mathematics

RATIONAL POLYNOMIALS OF SIMPLE TYPE

Walter D. Neumann and Paul Norbury 


\title{
RATIONAL POLYNOMIALS OF SIMPLE TYPE
}

\author{
Walter D. Neumann and Paul Norbury
}

\begin{abstract}
We classify two-variable polynomials which are rational of simple type. These are precisely the two-variable polynomials with trivial homological monodromy.
\end{abstract}

\section{Introduction.}

A polynomial map $f: \mathbb{C}^{2} \rightarrow \mathbb{C}$ is rational if its generic fibre, and hence every fibre, is of genus zero. It is of simple type if, when extended to a morphism $\widetilde{f}: X \rightarrow \mathbb{P}^{1}$ of a compactification $X$ of $\mathbb{C}^{2}$, the restriction of $\widetilde{f}$ to each curve $C$ of the compactification divisor $D=X-\mathbb{C}^{2}$ is either degree 0 or 1 . The curves $C$ on which $\widetilde{f}$ is non-constant are called horizontal curves, so one says briefly "each horizontal curve is degree 1".

The classification of rational polynomials of simple type gained some new interest through the result of Cassou-Nogues, Artal-Bartolo, and Dimca [4] that they are precisely the polynomials whose homological monodromy is trivial (it suffices that the homological monodromy at infinity be trivial by an observation of Dimca).

A classification appeared in [12], but it is incomplete. It implicitly assumes trivial geometric monodromy (on page 346, lines 10-11). Trivial geometric monodromy implies isotriviality (generic fibres pairwise isomorphic) and turns out to be equivalent to it for rational polynomials of simple type. The classification in the non-isotrivial case was announced in the final section of [17]. The main purpose of this paper is to prove it. But we recently discovered that there are also isotrivial rational polynomials that are not in [12], so we have added a classification for the isotrivial case using our methods. This case can also be derived from Kaliman's classification [9] of all isotrivial polynomials. The fact that his list includes rational polynomials of simple type that are not in [12] appears not to have been noticed before (it also includes rational polynomials not of simple type).

In general, the classification of polynomial maps $f: \mathbb{C}^{2} \rightarrow \mathbb{C}$ is an open problem with extremely rich structure. One notable result is the theorem of Abhyankar-Moh and Suzuki $[\mathbf{1}, \mathbf{2 3}]$ which classifies all polynomials with one fibre isomorphic to $\mathbb{C}$. The analogous result for the next simplest case, where one fibre is isomorphic to $\mathbb{C}^{*}$, is open except in special cases where the 
genus of the generic fibre of the polynomial is given. Kaliman [10] classifies all rational polynomials with one fibre isomorphic to $\mathbb{C}^{*}$.

The basic tool we use in our study of rational polynomials is to associate to any rational polynomial $f: \mathbb{C}^{2} \rightarrow \mathbb{C}$ a compactification $X$ of $\mathbb{C}^{2}$ on which $f$ extends to a well-defined map $\widetilde{f}: X \rightarrow \mathbb{P}^{1}$ together with a map $X \rightarrow \mathbb{P}^{1} \times \mathbb{P}^{1}$. The map to $\mathbb{P}^{1} \times \mathbb{P}^{1}$ is not in general canonical. We will exploit the fact that for a particular class of rational polynomials, there is an almost canonical choice.

Although we give explicit polynomials, the classification is initially presented in terms of the splice diagram for the link at infinity of a generic fibre of the polynomial (Theorem 4.1). This is called the regular splice diagram for the polynomial (since generic fibres are also called "regular"). See [15] for a description of the link at infinity and its splice diagram. The regular splice diagram determines the embedded topology of a generic fibre and the degree of each horizontal curve. Hence we can speak of a "rational splice diagram of simple type".

The first author has asked if the moduli space of polynomials with given regular splice diagram is connected. For a rational splice diagram of simple type we find the answer is "yes". We describe the moduli space for our polynomials in Theorem 4.2 and use it to help give explicit normal forms for the polynomials. We also describe how the topology of the irregular fibres varies over the moduli space.

The more general problem of classifying all rational polynomials, which would cover much of the work mentioned above, is still an open and interesting problem. It is closely related to the problem of classifying birational morphisms of the complex plane since a polynomial is rational if and only if it is one coordinate of a birational map of the complex plane. Russell [20] calls this a "field generator" and defines a good field generator to be a rational polynomial that is one coordinate of a birational morphism of the complex plane. A rational polynomial is good precisely when its resolution has at least one degree one horizontal curve, [20]. Daigle [5] studies birational morphisms $\mathbb{C}^{2} \rightarrow \mathbb{C}^{2}$ by associating to a compactification $X$ of the domain plane a canonical map $X \rightarrow \mathbb{P}^{2}$. A birational morphism is then given by a set of curves and points in $\mathbb{P}^{2}$ indicating where the map is not one-to-one. The approach we use in this paper is similar.

The full list of rational polynomials $f: \mathbb{C}^{2} \rightarrow \mathbb{C}$ of simple type is as follows. We list them up to polynomial automorphisms of domain $\mathbb{C}^{2}$ and range $\mathbb{C}$ (so-called "right-left equivalence"). 
Theorem 1.1. Up to right-left equivalence a rational polynomial $f(x, y)$ of simple type has one of the following forms $f_{i}(x, y), i=1,2$, or 3 .

$$
\begin{array}{ll}
f_{1}(x, y)=x^{q_{1}} s^{q}+x^{p_{1}} s^{p} \prod_{i=1}^{r-1}\left(\beta_{i}-x^{q_{1}} s^{q}\right)^{a_{i}} & \\
f_{2}(x, y)=x^{p_{1}} s^{p} \prod_{i=1}^{r-1}\left(\beta_{i}-x^{q_{1}} s^{q}\right)^{a_{i}} & (r \geq 1) \\
f_{3}(x, y)=y \prod_{i=1}^{r-1}\left(\beta_{i}-x\right)^{a_{i}}+h(x) & (r \geq 1) .
\end{array}
$$

Here:

$0 \leq q_{1}<q, \quad 0 \leq p_{1}<p, \quad\left|\begin{array}{cc}p & p_{1} \\ q & q_{1}\end{array}\right|= \pm 1 ;$

$s=y x^{k}+P(x)$, with $k \geq 1$ and $P(x)$ a polynomial of degree $<k$;

$a_{1}, \ldots, a_{r-1}$ are positive integers;

$\beta_{1}, \ldots, \beta_{r-1}$ are distinct elements of $\mathbb{C}^{*}$;

$h(x)$ is a polynomial of degree $<\sum_{1}^{r-1} a_{i}$.

Moreover, if $g_{1}(x, y)=g_{2}(x, y)=x^{q_{1}} s^{q}$ and $g_{3}(x, y)=x$ then $\left(f_{i}, g_{i}\right): \mathbb{C}^{2}$ $\rightarrow \mathbb{C}^{2}$ is a birational morphism for $i=1,2,3$. In fact, $g_{i}$ maps a generic fibre $f_{i}^{-1}(t)$ biholomorphically to $\mathbb{C}-\left\{0, t, \beta_{1}, \ldots, \beta_{r-1}\right\}, \mathbb{C}-\left\{0, \beta_{1}, \ldots, \beta_{r-1}\right\}$, or $\mathbb{C}-\left\{\beta_{1}, \ldots, \beta_{r-1}\right\}$, according as $i=1,2,3$. Thus $f_{1}$ is not isotrivial and $f_{2}$ and $f_{3}$ are.

In [12] the isotrivial case is subdivided into seven subcases, but these do not include any $f_{2}(x, y)$ with $p, q, p_{1}, q_{1}$ all $>1$.

\section{Resolution.}

Given a polynomial $f: \mathbb{C}^{2} \rightarrow \mathbb{C}$, extend it to a map $\bar{f}: \mathbb{P}^{2} \rightarrow \mathbb{P}^{1}$ and resolve the points of indeterminacy to get a regular map $\widetilde{f}: X \rightarrow \mathbb{P}^{1}$ that coincides with $f$ on $\mathbb{C}^{2} \subset X$. We call $D=X-\mathbb{C}^{2}$ the divisor at infinity. The divisor $D$ consists of a connected union of rational curves. An irreducible component $E$ of $D$ is horizontal if the restriction of $\widetilde{f}$ to $E$ is not a constant mapping. The degree of a horizontal curve $E$ is the degree of the restriction $\widetilde{f} \mid E$. Although the compactification defined above is not unique, the horizontal curves are essentially independent of choice.

Note that a generic fibre $F_{c}:=f^{-1}(c)$ is a punctured Riemann surface with punctures precisely where $\bar{F}_{c}$ meets a horizontal curve. Thus $f$ has simple type if and only if $\bar{F}_{c}$ meets each horizontal curve exactly once, so the number of punctures equals the number of horizontal curves. For nonsimple type the number of punctures will exceed the number of horizontal curves. 
We say that a rational polynomial is ample if it has at least three degree one horizontal curves. Those polynomials with no degree one horizontal curves, or bad field generators [20], are examples of polynomials that are not ample. The classification of Kaliman [10] mentioned in the introduction gives examples of polynomials with exactly one degree one horizontal curve so they are also not ample. Nevertheless, ample rational polynomials will be the focus of our study in this paper. We will classify all ample rational polynomials that are also of simple type.

\section{Curves in $\mathbb{P}^{1} \times \mathbb{P}^{1}$.}

If $\tilde{f}: X \rightarrow \mathbb{P}^{1}$ is a regular map with rational fibres then $X$ can be blown down to a Hirzebruch surface, $S$, so that $\widetilde{f}$ is given by the composition of the sequence of blow-downs $X \rightarrow S$ with the natural map $S \rightarrow \mathbb{P}^{1}$; see [2] for details. Moreover, by first replacing $X$ by a blown-up version of $X$ if necessary, we may assume that $S=\mathbb{P}^{1} \times \mathbb{P}^{1}$ and the natural map to $\mathbb{P}^{1}$ is projection onto the first factor.

A rational polynomial $f: \mathbb{C}^{2} \rightarrow \mathbb{C}$, once compactified to $\tilde{f}: X=\mathbb{C}^{2} \cup D \rightarrow$ $\mathbb{P}^{1}$, may thus be given by $\mathbb{P}^{1} \times \mathbb{P}^{1}$ together with instructions how to blow up $\mathbb{P}^{1} \times \mathbb{P}^{1}$ to get $X$ and how to determine $D$ in $X$. For this we give the following data:

- A collection $\mathcal{C}$ of irreducible rational curves in $\mathbb{P}^{1} \times \mathbb{P}^{1}$ including $L_{\infty}:=$ $\infty \times \mathbb{P}^{1}$

- a set of instructions on how to blow up $\mathbb{P}^{1} \times \mathbb{P}^{1}$ to obtain $X$;

- a sub-collection $\mathcal{E}$ of the curves of the exceptional divisor of $X \rightarrow$ $\mathbb{P}^{1} \times \mathbb{P}^{1}$

satisfying the condition:

- If $D$ is the union of the curves of $\mathcal{E}$ and the proper transforms of the curves of $\mathcal{C}$ then $X-D \cong \mathbb{C}^{2}$.

If $C \subset \mathbb{P}^{1} \times \mathbb{P}^{1}$ is an irreducible algebraic curve we associate to it the pair of integers $(m, n)$ given by degrees of the two projections of $C$ to the factors of $\mathbb{P}^{1} \times \mathbb{P}^{1}$. Equivalently, $(m, n)$ is the homology class of $C$ in terms of $H_{2}\left(\mathbb{P}^{1} \times \mathbb{P}^{1}\right)=\mathbb{Z} \oplus \mathbb{Z}$. We call $C$ an $(m, n)$ curve. The intersection number of an $(m, n)$ curve $C$ and an $\left(m^{\prime}, n^{\prime}\right)$ curve $C^{\prime}$ is $C \cdot C^{\prime}=m n^{\prime}+n m^{\prime}$.

The above collection $\mathcal{C}$ of curves in $\mathbb{P}^{1} \times \mathbb{P}^{1}$ will consist of some vertical curves (that is, $(0,1)$ curves; one of these is $\left.L_{\infty}\right)$ and some other curves. These non-vertical curves give the horizontal curves for $f$, so they all have $m=1$ if $f$ is of simple type. Note that a $(1, n)$ curve is necessarily smooth and rational (since it is the graph of a morphism $\mathbb{P}^{1} \rightarrow \mathbb{P}^{1}$ ).

The image in $\mathbb{P}^{1} \times \mathbb{P}^{1}$ of the fibre over infinity is the $(0,1)$ curve $L_{\infty}$ and the image of a degree $m$ horizontal curve is an $(m, n)$ curve. This view allows one to see as follows a geometric proof of the result of Russell [20] 
that a rational polynomial $f$ is good precisely when its resolution has at least one degree one horizontal curve. A degree one horizontal curve for $f$ has image in $\mathbb{P}^{1} \times \mathbb{P}^{1}$ given by a $(1, n)$ curve. Call this image $C$ and let $P$ be its intersection with $L_{\infty}$. The $(1, n)$ curves that do not intersect $C-P$ form a $\mathbb{C}$-family that sweeps out $\mathbb{P}^{1} \times \mathbb{P}^{1}-\left(L_{\infty} \cup C\right)$ so they lead to a map $X \rightarrow \mathbb{P}^{1}$ which takes values in $\mathbb{C}$ at points that do not lie over $L_{\infty} \cup C$. Restricting to $\mathbb{C}^{2}=X-D$ we obtain a meromorphic function $g_{1}$ that has poles only at points that belong to exceptional curves that were blown up on $C$ (and do not belong to $\mathcal{E}$ ). However the polynomial $f$ is constant on each such curve, so if $c_{1}, \ldots, c_{k}$ are the values that $f$ takes on these curves, then $g:=g_{1}\left(f-c_{1}\right)^{a_{1}} \ldots\left(f-c_{k}\right)^{a_{k}}$ will have no poles, and hence be polynomial, for $a_{1}, \ldots, a_{k}$ sufficiently large. Then $(f, g)$ is the desired birational morphism $\mathbb{C}^{2} \rightarrow \mathbb{C}^{2}$. For the converse, given a birational morphism $(f, g): \mathbb{C}^{2} \rightarrow \mathbb{C}^{2}$, we compactify it to a morphism $(\widetilde{f}, \widetilde{g}): X \rightarrow \mathbb{P}^{1} \times \mathbb{P}^{1}$. Then the proper transform of $\mathbb{P}^{1} \times \infty$ is the desired degree one horizontal curve for $f$.

We shall use the usual encoding of the topology of $D$ by the dual graph, which has a vertex for each component of $D$, an edge when two components intersect, and vertex weights given by self-intersection numbers of the components of $D$. We will sometimes speak of the valency of a component $C$ of $D$ to mean the valency of the corresponding vertex of the dual graph, that is, the number of other components that $C$ meets.

The approach we will take to get rational polynomials will be to start with any collection $\mathcal{C}$ of $k$ curves in $\mathbb{P}^{1} \times \mathbb{P}^{1}$ and see if we can produce a divisor at infinity $D$ for a map from $\mathbb{C}^{2}$ to $\mathbb{C}$. In order to get a divisor at infinity we must blow up $\mathbb{P}^{1} \times \mathbb{P}^{1}$, say $m$ times, and include some of the resulting exceptional curves in the collection so that this new collection gives a divisor $D$ whose complement is $\mathbb{C}^{2}$. The exceptional curves that we "leave behind" (i.e., do not include in $D$ ) will be called cutting divisors.

Lemma 3.1. $\quad$ (i) D must have $m+2$ irreducible components, so we must include $m-k+2$ of the exceptional divisors in the collection leaving $k-2$ behind as cutting divisors;

(ii) D must be connected and have no cycles;

(iii) D must reduce to one of the "Morrow configurations" by a sequence of blow-downs. The Morrow configurations are the configurations of rational curves with dual graphs of one of the following three types, in which, in the last case, after replacing the central $(n, 0,-n-1)$ by a single $(-1)$ vertex the result should blow down to a single $(+1)$ vertex by a sequence of blow-downs:

\begin{tabular}{|c|c|c|c|c|c|c|c|c|}
\hline & & & & & & & & \\
\hline & & & & 0 & $l$ & & & \\
\hline & & & & 0 & 0 & & & \\
\hline$l_{m}$ & $\ldots$ & $l_{1}$ & $n$ & 0 & $-n-1$ & $t_{1}$ & $\ldots$ & $t_{k}$ \\
\hline
\end{tabular}


These conditions are also sufficient that $X-D \cong \mathbb{C}^{2}$.

Proof. The first property follows from the fact that each blow-up increases the rank of second homology by 1 . Thus $H_{2}(X)$ has rank $m+2$, so $D$ must have $m+2$ irreducible components. Notice that this implies easily the well-known result $[\mathbf{1 1}, \mathbf{1 2}, \mathbf{2 3}]$ that

$$
\delta-1=\sum_{a \in \mathbb{C}}\left(r_{a}-1\right),
$$

where $\delta$ is the number of horizontal curves of $f$ and $r_{a}$ is the number of irreducible components of $f^{-1}(a)$. (Both sides are equal to $k-1-\{$ number of finite curves at infinity\}.)

The second property follows from the third property. For the third property and sufficiency see $[\mathbf{1 3}, \mathbf{1 9}]$.

Now assume that $\tilde{f}$ has at least three degree one horizontal curves. Take these three horizontal curves and use them to map $X$ to $\mathbb{P}^{1} \times \mathbb{P}^{1}$ as follows. The three horizontal curves define three points in a generic fibre of $\widetilde{f}$. We can map this generic fibre to $\mathbb{P}^{1}$ by mapping these three points to $0,1, \infty \in \mathbb{P}^{1}$. This defines a map from a Zariski open set of $X$ to $\mathbb{P}^{1}$ which then extends to a map $\pi$ from $X$ to $\mathbb{P}^{1}$. If $\pi$ is not a morphism then we blow up $X$ to get a morphism. Rather than introducing further notation for this blow-up we will assume we began with this blow-up and call it $X$. Together with the map $\tilde{f}$ this gives us the desired morphism

$$
X \stackrel{(\widetilde{f}, \pi)}{\longrightarrow} \mathbb{P}^{1} \times \mathbb{P}^{1}
$$

with the property that the three horizontal curves map to $(1,0)$ curves.

If all horizontal curves for $f$ are of type $(1,0)$ then the generic fibres form an isotrivial family (briefly " $f$ is isotrivial"). Thus if $f$ is of simple type but not isotrivial, there must be a horizontal curve of type $(1, n)$ in $\mathcal{C}$ with $n>0$. From now on, therefore, we assume that there are at least three $(1,0)$ curves and at least one $(1, n)$ curve in $\mathcal{C}$ with $n>0$.

Lemma 3.2. Any curve of $D$ that is beyond a horizontal curve from the point of view of $\widetilde{L}_{\infty}$ has self-intersection $\leq-2$.

Proof. If the curve is an exceptional curve then it has self-intersection $\leq$ -1 . If -1 , then the curve must have valency at least three (since any -1 exceptional curve that could be blown down is a cutting divisor). Any three adjacent curves must include two horizontal curves, which contradicts the fact that the dual graph of $D$ has no cycles. If the curve is not exceptional then it is the proper transform of a vertical curve. But we must have blown up at least three times on the vertical curve to get rid of cycles in the dual graph of $D$ so in this case the self-intersection is $\leq-3$. 
3.1. Horizontal curves. The next few lemmas will be devoted to finding restrictions on the horizontal curves in the configuration $\mathcal{C} \subset \mathbb{P}^{1} \times \mathbb{P}^{1}$, culminating in Proposition 3.9.

Lemma 3.3. A horizontal curve of type $(1, n)$ in $\mathcal{C}$ must be of type $(1,1)$.

Proof. Assume we have a horizontal curve $C \in \mathcal{C}$ of type $(1, n)$ with $n>1$. It intersects each of the three $(1,0)$ curves $n$ times (counting with multiplicity) so in order to break cycles - Lemma 3.1 (ii) - we have to blow up at least $n$ times on each $(1,0)$ horizontal curve, so the proper transforms of the three $(1,0)$ curves have self-intersection at most $-n$ and the proper transform of the $(1, n)$ curve has self-intersection at most $2 n-3 n=-n$.

By Lemma 3.1 (iii), $D$ must reduce to a Morrow configuration by a sequence of blow-downs. Thus $D$ must contain a -1 curve $E$ that blows down. By Lemma 3.2, the curve $E$ must be a proper transform of a horizontal curve. The proper transform of each $(1,0)$ curve has self-intersection at most $-n<-1$. Thus $E$ must come from one of the $(1, *)$ horizontal curves. As mentioned above, the proper transform of a $(1, k)$ curve has self-intersection $\leq-k$ so $E$ must be the proper transform of a $(1,1)$ curve, $E_{0}$. But $E_{0}$ would intersect $C$, the $(1, n)$ curve, $2 n$ times and hence $E . E \leq 2-2 n<-1$ since $n>1$. This is a contradiction so any horizontal curve of type $(1, n)$ must be a $(1,1)$ curve.

Hence, the horizontal curves consist of a collection of $(1,0)$ curves and $(1,1)$ curves. Figure 1 shows an example of a possible configuration of horizontal curves in $\mathbb{P}^{1} \times \mathbb{P}^{1}$.

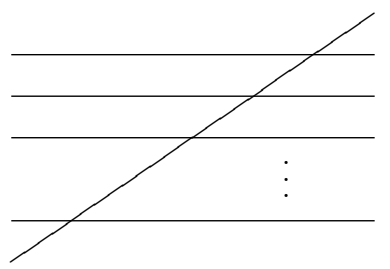

Figure 1. Configuration of horizontal curves.

Lemma 3.4. $\widetilde{L}_{\infty} \cdot \widetilde{L}_{\infty}=-1$.

Proof. We blow up at a point on $L_{\infty}$ precisely when at least two horizontal curves meet in a common point there. In general, if a horizontal curve meets $L_{\infty}$ with a high degree of tangency then we blow up repeatedly there. But, since all horizontal curves are $(1,0)$ and $(1,1)$ curves, they meet $L_{\infty}$ transversally, so a point on $L_{\infty}$ will be blown up at most once. 
If there are two such points to be blown up, then after blowing up there will be (in the dual graph) two non-neighbouring -1 curves with valency $>2$. The complement of such a configuration cannot be $\mathbb{C}^{2}$. This is proven by Kaliman [11] as Corollary 3. Actually the result is stated for two -1 curves of valency 3 but it applies to valency $\geq 3$.

Thus, at most one point on $L_{\infty}$ is blown up and $\widetilde{L}_{\infty} \cdot \widetilde{L}_{\infty}=0$ or -1 . We must show 0 cannot occur.

Since there are at least four horizontal curves, if $\widetilde{L}_{\infty} \cdot \widetilde{L}_{\infty}=0$, then $\widetilde{L}_{\infty}$ has valency at least 4 and every other curve has negative self-intersection. Furthermore, the only possible -1 curves must be horizontal curves, and these intersect $\widetilde{L}_{\infty}$ in $D$. As we attempt to blow down $D$ to get to a Morrow configuration, the only curves that can be blown down will always be adjacent to $\widetilde{L}_{\infty}$. Thus the intersection number of $\widetilde{L}_{\infty}$ will become positive and all other intersection numbers remain negative, so a Morrow configuration cannot be reached. Hence, $\widetilde{L}_{\infty} \cdot \widetilde{L}_{\infty}=-1$.

Lemma 3.5. A configuration of curves that contains two branches consisting of curves of self-intersection $<-1$ that meet at a valency $>2$ curve of self-intersection greater than or equal to -1 as in Figure 2 (where the meeting curve is drawn with valency 3 for convenience) cannot be blown down to a Morrow configuration.

Proof. Since the two branches consist of curves of self-intersection $<-1$, they cannot be reduced before the other branches are reduced. If the rest of the configuration of curves is blown down first then the valency $>2$ curve becomes a valency 2 curve with non-negative self-intersection and no more blow-downs can be done. Since there is no 0 curve, we have not reached a Morrow configuration.

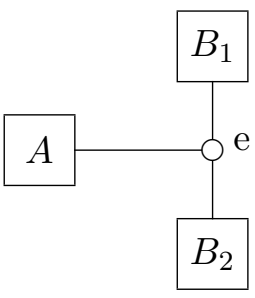

Figure 2. The branches $B_{1}$ and $B_{2}$ consist of curves of selfintersection $<-1$ and $e \geq-1$.

Lemma 3.6. The intersection of any two $(1,1)$ curves in $\mathcal{C}$ consists of two distinct points contained in the union of the $(1,0)$ curves in $\mathcal{C}$. 
Proof. We will assume otherwise and reduce to the situation of Lemma 3.5 to give a contradiction. Thus, assume that two $(1,1)$ curves do not intersect in two points contained in the union of the $(1,0)$ curves. Then in order to break cycles these curves must be blown up at least four times - once each for at least three of the $(1,0)$ curves and at least another time for the intersection of the two $(1,1)$ curves. Thus they have self-intersection $<-1$.

Case 1: Suppose two $(1,1)$ curves meet on $L_{\infty}$. Then after blowing up (twice if the $(1,1)$ curves meet at a tangent), the exceptional curves are retained and the final exceptional curve has self-intersection -1 , valency 3 and two branches, which we will call $B_{1}$ and $B_{2}$, consisting of the proper transforms of the two $(1,1)$ curves and any other curves beyond these proper transforms all of which have self-intersection $<-1$. Thus we are in the situation of Lemma 3.5 and we get a contradiction.

Case 2: Suppose two $(1,1)$ curves meet $L_{\infty}$ at distinct points. Then at least one of the $(1,1)$ curves, $D$, must meet $L_{\infty}$ at a point away from the $(1,0)$ curves by Lemma 3.4. Also one of the $(1,0)$ curves, $H$, must meet $L_{\infty}$ away from the $(1,1)$ curves and contain at least two points where it intersects the $(1,1)$ curves and thus have self-intersection $<-1$ after blowing up to break cycles. We are once more at the situation of Lemma 3.5 where the valency $>2$ curve is $\widetilde{L}_{\infty}$ which has self-intersection -1 by Lemma 3.4 , and the branches $B_{1}$ and $B_{2}$ are the proper transform of $D$ and any curves beyond it, respectively the proper transform of $H$ and any curves beyond it. Thus we have a contradiction.

Notice that both cases apply to two $(1,1)$ curves that may intersect at a tangent point, and shows that this situation is impossible.

Lemma 3.7. If there is more than one $(1,1)$ curve in $\mathcal{C}$ then there are exactly three $(1,0)$ horizontal curves in $\mathcal{C}$.

Proof. Assume that there are more than three $(1,0)$ horizontal curves in $\mathcal{C}$ and at least two $(1,1)$ curves, say $C_{1}$ and $C_{2}$.

Case 1: $C_{1}$ and $C_{2}$ meet on $\widetilde{L}_{\infty}$. Then they meet each of at least two $(1,0)$ curves in distinct points, so after blowing up to destroy cycles, these $(1,0)$ curves have self-intersection $\leq-2$ and Lemma 3.5 applies.

Case 2: $C_{1}$ and $C_{2}$ meet $\widetilde{L}_{\infty}$ at distinct points. Then one of them, say $C_{1}$, meets $\widetilde{L}_{\infty}$ at a point not on a $(1,0)$ curve by Lemma 3.4 . At least one $(1,0)$ curve $C_{3}$ meets $C_{1}$ and $C_{2}$ in distinct points. After breaking cycles, $C_{1}$ and $C_{3}$ have self-intersections $\leq-2$ so Lemma 3.5 applies again.

Lemma 3.8. A family of $(1,1)$ horizontal curves in $\mathcal{C}$ must pass through a common pair of points. 
Proof. The statement is trivial for one $(1,1)$ horizontal curve so assume there are at least two $(1,1)$ horizontal curves in $\mathcal{C}$. By the previous lemma, there are exactly three $(1,0)$ horizontal curves.

If there are exactly two $(1,1)$ horizontal curves in $\mathcal{C}$ then the lemma is clear since the curves cannot be tangent by Lemma 3.6.

When there are more than two $(1,1)$ curves in $\mathcal{C}$, apply Lemma 3.6 to two of them. If another $(1,1)$ horizontal curve in $\mathcal{C}$ does not intersect these two $(1,1)$ curves at their common two points of intersection then, by Lemma 3.6, it must meet both these $(1,1)$ curves at the third $(1,0)$ horizontal curve of $\mathcal{C}$. So the first two $(1,1)$ curves would meet there, which is a contradiction.

Proposition 3.9. Any configuration of horizontal curves in $\mathcal{C}$ is equivalent to one of the form in Figure 1.

Proof. By assumption and Lemma 3.3 there are at least three $(1,0)$ horizontal curves and some $(1,1)$ horizontal curves in $\mathcal{C}$. If there is exactly one $(1,1)$ horizontal curve then the proposition is clear. If there is more than one $(1,1)$ horizontal curve, then by Lemmas 3.7 and 3.8 there are precisely three $(1,0)$ horizontal curves and two of the $(1,0)$ horizontal curves contain the common intersection of the $(1,1)$ curves. Each $(1,1)$ curve also contains a distinguished point where the curve meets the third $(1,0)$ horizontal curve. A Cremona transformation can bring such a configuration to that in Figure 1 by blowing up at the two points of intersection of the $(1,1)$ curves and blowing down the two vertical lines containing the two points. This sends two of the $(1,0)$ horizontal curves and each $(1,1)$ curve to $(1,0)$ horizontal curves and one of the $(1,0)$ curves to a $(1,1)$ curve that intersects each of the other horizontal curves exactly once. Note that since we blow up $\mathbb{P}^{1} \times \mathbb{P}^{1}$ to get the polynomial map, two configurations of curves $\mathcal{C}, \mathcal{C}^{\prime}$ in $\mathbb{P}^{1} \times \mathbb{P}^{1}$ related by a Cremona transformation give rise to the same polynomial, so we are done.

3.2. The configuration $\mathcal{C}$. The image $\mathcal{C}$ of $D \subset X \rightarrow \mathbb{P}^{1} \times \mathbb{P}^{1}$ will consist of the configuration of horizontal curves in Figure 1 plus some $(0,1)$ vertical curves. The next two lemmas show that in fact the only $(0,1)$ vertical curve we need to include in $\mathcal{C}$ is $L_{\infty}$ and furthermore that $\mathcal{C}$ can be given by Figure 4 .

\section{Lemma 3.10. The configuration $\mathcal{C}$ appears in Figure 3 or Figure 4.}

Proof. Let $r+2$ denote the number of horizontal curves and $k+1$ denote the number of $(0,1)$ vertical curves in $\mathcal{C}$. Thus $\mathcal{C}$ consists of $k+r+3$ irreducible components and by Lemma 3.1 (i), when blowing up to get $D$ from $\mathcal{C}$ we must leave $k+r+1$ exceptional curves behind as cutting divisors.

By Lemma 3.1 (ii) we must break all cycles. The minimum number of cutting divisors needed to do this is $k r+k+r-2 \min \{k, r\}$. This is because each of the $k(0,1)$ vertical curves different from $L_{\infty}$ must be separated from 
all but one of the $r+1(1,0)$ horizontal curves, so we need $k r$ cutting divisors. Also, the $(1,1)$ horizontal curve meets each of the $r+1(1,0)$ horizontal curves and each of the $k(0,1)$ vertical curves once, so that requires $k+r$ cutting divisors (by Lemma 3.4 the $(1,1)$ curve must meet $L_{\infty}$ at a triple point with a $(1,0)$ horizontal curve, so this intersection does not produce a cycle to be broken). We would thus require $k r+k+r$ cutting divisors except that the $(1,1)$ curve may pass through intersections of the $(1,0)$ horizontal curves and the $(0,1)$ vertical curves, so some of the cutting divisors may coincide. The most such intersections possible is $\min \{k, r\}$ and we have then over-counted required cutting divisors by $2 \min \{k, r\}$. Hence we get at least $k r+k+r-2 \min \{k, r\}$ cutting divisors.

Since the number $k+r+1$ of cutting divisors is at least $k r+k+r-$ $2 \min \{k, r\}$, we have $k+r+1 \geq k r+k+r-2 \min \{k, r\}$, so

$$
1 \geq k(r-2) \text { and } 1 \geq(k-2) r, \quad k \geq 0, r \geq 2 .
$$

The solutions of $(1)$ are $(k, r)=\{(0, r),(1,2),(1,3),(2,2)\}$.

Recall by Lemma 3.4 that the $(1,1)$ curve must meet $L_{\infty}$ at a triple point with a $(1,0)$ horizontal curve. Furthermore, by keeping track of when either inequality in (1) is an equality, or one away from an equality, we can see that the $(1,1)$ curve must meet any other $(0,1)$ vertical curves at a triple point with a $(1,0)$ horizontal curve. Thus, the only possible configurations for $\mathcal{C}$ are given in Figures 3 and 4.
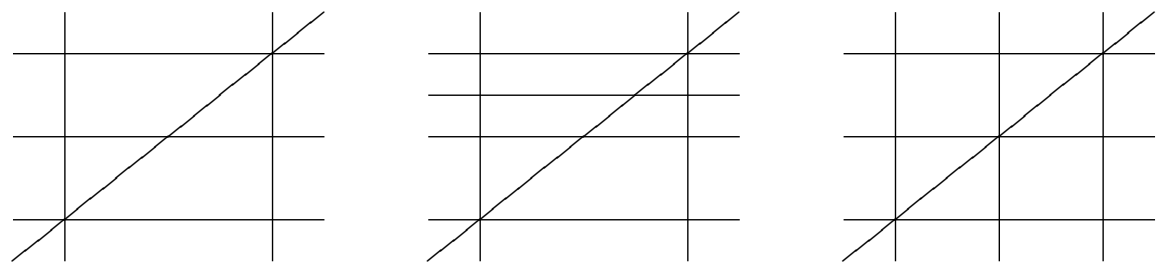

Figure 3. Configuration $\mathcal{C}$.



Figure 4. Configuration $\mathcal{C}$ with $r+2$ horizontal curves. 
In the following lemmas we will exclude the configurations in Figure 3. Label the triple points in the first two configurations of Figure 3 by $P_{\infty} \in$ $L_{\infty}$ and $P_{1}$, and in the third configuration by $P_{\infty}, P_{1}, P_{2}$. Also, label the exceptional divisor obtained by blowing up the triple point $P_{i}$ by $E_{i}$ and its proper transform by $\widetilde{E}_{i}$.

Lemma 3.11. If $E_{i}$ is a cutting divisor then the $(0,1)$ vertical curve containing $P_{i}$ can be removed from $\mathcal{C}$ by a birational transformation.

Proof. In each of the configurations of Figure 3 we can perform a Cremona transformation by blowing up $P_{\infty}$ and $P_{i}$ for $i=1$ or 2 and then blowing down $\widetilde{L}_{\infty}$ and the proper transform of the $(0,1)$ vertical curve that contains $P_{i}$. The exceptional divisors $E$ and $E_{i}$ become $(0,1)$ curves and the $(0,1)$ vertical curve that contains $P_{i}$ becomes an exceptional divisor in a new configuration $\mathcal{C}$. When $E_{i}$ is a cutting divisor this operation essentially removes a $(0,1)$ vertical curve from $\mathcal{C}$.

Lemma 3.12. In a configuration from Figure 3 with $(k, r) \in\{(1,3),(2,2)\}$ at least one of the exceptional divisors $E_{1}$ or $E_{2}$ is a cutting divisor.

Proof. Suppose otherwise, that $E_{1}$ is not a cutting divisor and for $(k, r)=$ $(2,2)$ nor is $E_{2}$ a cutting divisor. The exceptional curves $E_{i}$ introduce an extra intersection and hence an extra cutting divisor is required. There is one such extra intersection in the configuration with $(k, r)=(1,3)$ and two such extra intersections in the configuration with $(k, r)=(2,2)$. As mentioned in the proof of Lemma 3.10 the solution $(k, r)=(1,3)$ gives equality in (1) and so it cannot sustain an extra cutting divisor. Similarly the solution $(k, r)=(2,2)$ is 1 away from equality in (1) and so it cannot sustain two extra cutting divisors. Hence we get a contradiction and the lemma is proven.

By the previous two lemmas we can simplify any configuration from Figure 3 to lie in Figure 4 or to be the first configuration from Figure 3 (the one with $(k, r)=(1,2))$ with the requirement that $E_{1}$ is not a cutting divisor. It is this last case that we will now exclude.

The next three lemmas suppose that we have the first configuration from Figure 3 and that $E_{1}$ is not a cutting divisor. We will denote the four horizontal curves by $H_{i}, i=1, \ldots, 4$, and their proper transforms by $\widetilde{H}_{i}$ where $H_{4}$ is the $(1,1)$ curve, $H_{1}$ contains $P_{1}$ and $H_{3}$ contains $P_{\infty}$. Also denote the $(1,0)$ vertical curve that contains $P_{1}$ by $L_{1}$ and its proper transform by $\widetilde{L}_{1}$.

Lemma 3.13. At least one of $\widetilde{H}_{1}$ and $\widetilde{H}_{2}$ and at least one of $\widetilde{H}_{3}$ and $\widetilde{H}_{4}$ has self-intersection -1 .

Proof. The proper transform of each horizontal curve has self-intersection less than or equal to -1 and all curves in $D$ beyond horizontal curves have 
self-intersection strictly less than -1 . If the two horizontal curves that meet $\widetilde{L}_{\infty}, \widetilde{H}_{1}$ and $\widetilde{H}_{2}$, have self-intersection strictly less than -1 , then since all curves beyond the two horizontal curves also have self-intersection strictly less than -1 , and since $\widetilde{L}_{\infty}$ has self-intersection -1 and valence 3 this gives a contradiction by Lemma 3.5. The same argument applies to $\widetilde{H}_{3}$ and $\widetilde{H}_{4}$ together with $E$.

Lemma 3.14. $\widetilde{H}_{4} \cdot \widetilde{H}_{4}=-1$ if and only if $\widetilde{H}_{2} \cdot \widetilde{H}_{2}=-1$.

Proof. Since $L_{1}$ must be separated from at least one of $H_{2}$ and $H_{3}$ then at most one of $\widetilde{H}_{2} \cdot \widetilde{H}_{2}=-1$ and $\widetilde{H}_{3} \cdot \widetilde{H}_{3}=-1$ can be true. Similarly $E_{1}$ must be separated from at least one of $H_{1}$ and $H_{4}$ so at most one of $\widetilde{H}_{1} \cdot \widetilde{H}_{1}=-1$ and $\widetilde{H}_{4} \cdot \widetilde{H}_{4}=-1$ can be true. By Lemma 3.13 , if $\widetilde{H}_{2} \cdot \widetilde{H}_{2} \neq-1$ then $\widetilde{H}_{1} \cdot \widetilde{H}_{1}=-1$ so $\widetilde{H}_{4} \cdot \widetilde{H}_{4} \neq-1$. Similarly, $\widetilde{H}_{1} \cdot \widetilde{H}_{1} \neq-1$ implies that $\widetilde{H}_{2} \cdot \widetilde{H}_{2}=-1$ and $\widetilde{H}_{4} \cdot \widetilde{H}_{4}=-1$.

Lemma 3.15. The configuration from Figure 3 with $(k, r)=(1,2)$ together with the requirement that $E_{1}$ is not a cutting divisor cannot occur.

Proof. Suppose otherwise. Assume that $\widetilde{H}_{1} \cdot \widetilde{H}_{1}=-1$ and $\widetilde{H}_{3} \cdot \widetilde{H}_{3}=-1$. If this is not the case, then by Lemmas 3.13 and 3.14 we may assume that $\widetilde{H}_{4} \cdot \widetilde{H}_{4}=-1$ and $\widetilde{H}_{2} \cdot \widetilde{H}_{2}=-1$ and argue similarly. The curves beyond $\widetilde{H}_{1}$ have self-intersection strictly less than -1 . The curve immediately adjacent and beyond $\widetilde{H}_{1}$ is $\widetilde{E}_{1}$ and this has self-intersection strictly less than -2 . This is because we must blow up between $E_{1}$ and $H_{4}$ to separate cycles, and also between $\widetilde{E}_{1}$ and $\widetilde{L}_{1}$ to break cycles and to maintain $\widetilde{H}_{1} \cdot \widetilde{H}_{1}=$ -1 and $\widetilde{H}_{3} \cdot \widetilde{H}_{3}=-1$. Thus if we blow down $\widetilde{H}_{1}$ the remaining branch beyond $\widetilde{L}_{\infty}$ consists of curves with self-intersection strictly less than -1 . Also $\widetilde{H}_{2}$ has self-intersection strictly less than -1 since we have to blow up the intersection between $H_{2}$ and $H_{4}$ and the intersection between $H_{2}$ and $L_{1}$ in order to break cycles and maintain $\widetilde{H}_{3} \cdot \widetilde{H}_{3}=-1$. After blowing down $\widetilde{H}_{1}, \widetilde{L}_{\infty}$ has self-intersection 0 and valency 3 with two branches consisting of curves of self-intersection strictly less than -1 . Thus we can use Lemma 3.5 to get a contradiction.

\section{Non-isotrivial rational polynomials of simple type.}

The configuration in Figure 4 is the starting point for any non-isotrivial rational polynomial of simple type. Notice that we can fill one puncture in each fibre of any such map to get an isotrivial family of curves and the puncture varies linearly with $c \in \mathbb{C}$. Notice also that there is an irregular fibre for each of the $r$ intersection points of the $(1,1)$ curve with $(1,0)$ horizontal curves away from $L_{\infty}$. In fact there is at most one more irregular fibre which can only occur in rather special cases, as we discuss in Subsection 4.1. 
From now on the configuration $\mathcal{C}$ is given by Figure 4 with $r+2$ horizontal curves. Beginning with $\mathcal{C}$ we will list all of the rational polynomials of simple type generated from this configuration. We shall give the splice diagrams for these polynomials first. Although we compute the polynomials later, geometric information of interest is often more easily extracted from the splice diagram or from our construction of the polynomials than from an actual polynomial.

The splice diagram encodes the topology of the polynomial. It represents the link at infinity of the generic fibre, or it can be thought of as an efficient plumbing graph for the divisor at infinity, $D \subset X$. It encodes an entire parametrised family of polynomials with the same topology of their regular fibres. See $[\mathbf{7}, \mathbf{1 5}, \mathbf{1 6}]$ for more details. Within this family, polynomials can still differ in the topology of their irregular fibres. Our methods also give all information about the irregular fibres, as we describe in Subsection 4.1.

The configuration $\mathcal{C}$ has $r+3$ irreducible components so when we blow up to get $D$ by Lemma 3.1 (i) we will leave $r+1$ exceptional curves behind as cutting divisors. By Lemma 3.1 (ii) we must break the $r$ cycles in $\mathcal{C}$ with multiple blow-ups at the points of intersection leaving $r$ exceptional curves behind as cutting divisors. We blow up multiple times between the $r$ th $(1,0)$ horizontal curve and the $(1,1)$ horizontal curve in order to break a cycle. Thus, we require those blow-ups to satisfy the condition that the exceptional curve will break the cycle if removed. Equivalently, each new blow-up takes place at the intersection of the most recent exceptional curve with an adjacent curve. We call such a multiple blow-up a separating blow-up sequence.

We have one extra cutting divisor. This will arise as the last exceptional curve blown up in a sequence of blow-ups that does not break a cycle. We will call this sequence of blow-ups a non-separating blow-up sequence. A priori, this non-separating blow-up sequence could be a sequence as in Figure 5, where the final -1 curve is the cutting divisor. However, we shall

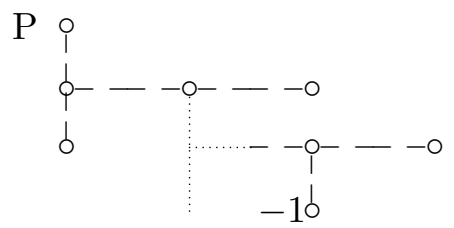

Figure 5. Sequence of blow-ups starting at $P$ and ending at the -1 curve.

see that the extra nodes this introduces in the dual graph prohibit $D$ from blowing down to a Morrow configuration, so the sequence is simply a string of -2 exceptional curves followed by -1 exceptional curve that is the cutting 
divisor. This arises from blowing up a point on a curve in the blow-up of $\mathcal{C}$ that does not lie on an intersection of irreducible components.

Let us begin by just performing the separating blow-up sequences at the points of intersection of $\mathcal{C}$ and leaving the non-separating blow-up sequence until later. This gives the dual graph in Figure 6 with the proper transforms of the $r+1(1,0)$ horizontal curves and the $(1,1)$ horizontal curve indicated along with $\widetilde{L}_{\infty}$ and the exceptional curve $E$ arising from the blow-up of the triple point in $\mathcal{C}$. There are $r$ branches heading out from the proper transform of $(1,1)$ consisting of curves of self-intersection less than -1 and beyond each of the proper transforms of the $r(1,0)$ horizontal curves the curves have self-intersection less than -1 .

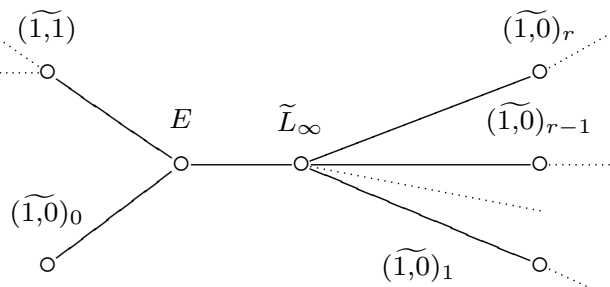

Figure 6. Dual graph of $\mathcal{C}$ blown up at points of intersection.

The self-intersection of each of $(\widetilde{1,0})_{0}, E$ and $\widetilde{L}_{\infty}$ is -1 . The self-intersections of $(\widetilde{1,1})$ and $(\widetilde{1,0})_{i}, i=1, \ldots, r$ are negative and depend on how we blow up at each point of intersection.

Lemma 4.1. There is at most one branch in $D$ beyond $(\widetilde{1,1})$, and $r-1$ of the horizontal curves $(\widetilde{1,0})_{i}$ (those with index $i=1, \ldots, r-1$ say) have self-intersection -1 and only -2 curves beyond.

Proof. Since the self-intersection of each of the curves beyond $(\widetilde{1,1})$ is less than -1 each branch beyond $(\widetilde{1,1})$ cannot be blown down before $(\widetilde{1,1})$. Thus, there are at most two branches.

Furthermore, since the self-intersection of each of the curves beyond $(\widetilde{1,0})_{i}$, $i=1, \ldots, r$ is less than -1 , the branch beyond $(\widetilde{1,0})_{i}$ can be blown down before $(\widetilde{1,0})_{i}$ only if $(\widetilde{1,0})_{i}$ has self-intersection -1 and each curve beyond has self-intersection -2 . Thus, at most two branches beyond $(\widetilde{1,0})_{i}, i=1, \ldots, r$ do not consist of a -1 curve with a string of -2 curves beyond. If there are two such branches then the blow-ups that create them create corresponding branches beyond $(\widetilde{1,1})$ (or possibly just decrease the intersection number at $(\widetilde{1,1})$. These two branches cannot be fully blown down until everything else connecting to the $\widetilde{L}_{\infty}$ vertex are blown down, but the vertex $(\widetilde{1,1})$ and any branches beyond it cannot blow down first. Thus $D$ cannot blow down 
to a Morrow configuration. Thus there is at most one such branch, proving the Lemma.

Figure 7 gives the dual graph of the partially blown up $\mathcal{C}$ where the label of each curve is now its self-intersection number. The branch beyond $(\widetilde{1,0})_{i}$ consists of a string of $a_{i}-1-2$ curves and $A=\sum_{i=1}^{r-1} a_{i}$. We have thus far only blown up once between the $r$ th $(1,0)$ horizontal curve and the $(1,1)$ horizontal curve, indicating the exceptional divisor by $\otimes$. We may blow up many more times - perform a separating blow-up sequence - leaving behind the final exceptional curve as cutting divisor to get a branch beyond $(\widetilde{1,1})$ and a branch beyond $(\widetilde{1,0})_{r}$. In addition, we still have to perform the nonseparating blow-up sequence at some point on the divisor.



Figure 7. Dual graph of partially blown-up configuration of curves.

Lemma 4.2. The non-separating blow-up sequence occurs beyond either $(\widetilde{1,1})$, $(\widetilde{1,0})_{r}$, or $(\widetilde{1,0})_{0}$ and in the latter case $(\widetilde{1,1}) \cdot(\widetilde{1,1})=-1$.

Proof. If the non-separating blow-up sequence occurs on the branch beyond $(\widetilde{1,0})_{i}, i=1, \ldots, r-1$ then that branch cannot be blown down. By the proof of Lemma 4.1, in order to obtain a linear graph we must blow down $r-1$ of the branches beyond $(\widetilde{1,0})_{i}, i=1, \ldots, r$. Thus, if the non-separating blowup sequence does occur beyond $(\widetilde{1,0})_{i}$ for some $i \leq r-1$, then the $(\widetilde{1,0})_{r}$ branch blows down, so we simply swap the labels $i$ and $r$.

The non-separating blow-up sequence cannot occur on $E$ or $\widetilde{L}_{\infty}$ because the resulting cutting divisor would not be sent to a finite value.

If the non-separating blow-up sequence occurs on the branch beyond $(\widetilde{1,0})_{0}$ then we must be able to blow down the branch beyond $(\widetilde{1,1})$, hence the branch must consist of $(\widetilde{1,1})$ with self-intersection -1 .

Lemma 4.3. We may assume the non-separating blow-up sequence does not occur beyond $(\widetilde{1,0})_{0}$. 
Proof. By Lemma 4.2 if the non-separating blow-up sequence occurs beyond $(\widetilde{1,0})_{0}$ then $(\widetilde{1,1}) \cdot(\widetilde{1,1})=-1$. In particular, $1=A=\sum_{1}^{r-1} a_{i}$. Thus, $r=2, a_{1}=1$. With only four horizontal curves, we can perform a Cremona transformation to make $(\widetilde{1,0})_{0}$ the $(1,1)$ curve and hence we are in the first case of Lemma 4.2.

Lemma 4.4. The non-separating blow-up sequence occurs on either of the last curves beyond $(\widetilde{1,1})$ or $(\widetilde{1,0})_{r}$ and is a string of -2 curves followed by the -1 curve that is a cutting divisor.

Proof. Arguing as previously, if the non-separating blow-up sequence occurs anywhere else, or if it is more complicated, then it introduces a new branch preventing the divisor $D$ from blowing down to a linear graph.

We now know that our divisor $D$ results from Figure 7 by doing a separating blow-up sequence between the $(1,1)$ curve and the $r$-th $(1,0)$ curve, leaving behind the final -1 exceptional curve as a cutting divisor and then performing a non-separating blow-up sequence on a curve adjacent to this cutting divisor to produce second cutting divisor.

A priori, it is not clear that this procedure always gives rise to a divisor $D \subset X$ where $X$ is a blow-up of $\mathbb{P}^{2}$ and $D$ is the pre-image of the line at infinity. The classification will be complete once we show it does.

Lemma 4.5. The above procedure always gives rise to a configuration that blows down to a Morrow configuration (see Lemma 3.1) and hence determines a rational polynomial of simple type.

Proof. The calculation involves the relation between plumbing graphs and splice diagrams described in [7] or [16], with which we assume familiarity. In particular, we use the continued fractions of weighted graphs described in [7]. If one has a chain of vertices with weights $-c_{0},-c_{1}, \ldots,-c_{t}$, its continued fraction based at the first vertex is defined to be

$$
c_{0}-\frac{1}{c_{1}-\frac{1}{c_{2}-\ddots}-\frac{1}{c_{t}}} .
$$

The dual graph for the curve configuration of Lemma 4.5 has chains starting at the vertex $(\widetilde{1,1})$ and $(\widetilde{1,0})_{r}$. We claim these chains have continued fractions evaluating to $A-1+\frac{P}{Q}$ and $\frac{q}{p}$ respectively, where $P, Q, p, q$ are arbitrary positive integers with $P q-p Q=1$. We describe the main ingredients of this calculation but leave the details to the reader.

An easy induction shows that the initial separating blow-up sequence leads to chains at $(\widetilde{1,1})$ and $(\widetilde{1,0})_{r}$ with continued fractions $A-1+\frac{n}{m}$ and 
$\frac{m}{n}$ with positive coprime $n$ and $m$. The non-separating blow-up sequence then changes the fraction $\frac{n}{m}$ or $\frac{m}{n}$ that it operates on as follows. If the nonseparating blow-up sequence consists of $k$ blow-ups at the end of the left chain then $\frac{n}{m}$ is replaced by $\frac{N}{M}$ with $N m-n M=1$ and $k \leq \frac{M}{m}<\frac{N}{n} \leq(k+1)$. If the non-separating blow-up sequence is on the right then $\frac{m}{n}$ is similarly changed instead.

Renaming, we can describe this in terms of our chosen names $p, q, P, Q$ as follows. We either have $P>p$ or $q>Q$. If $P>p$ the initial separating blow-up sequence leads to chains with continued fractions $A-1+\frac{p}{q}$ and $\frac{q}{p}$ and the non-separating blow-up sequence then consists of a sequence of $k:=\left\lfloor\frac{Q}{q}\right\rfloor$ blowups extending the left chain (and changing its continued fraction to $\left.A-1+\frac{P}{Q}\right)$. If $q>Q$ the continued fractions are $A-1+\frac{P}{Q}$ and $\frac{Q}{P}$ after the separating blowup and the non-separating blow-up consists of $k:=\left\lfloor\frac{p}{P}\right\rfloor$ blow-ups extending the right chain (and changing its continued fraction to $\left.\frac{q}{p}\right)$.

To prove the Lemma we must show that the dual graph of our curve configuration blows down to a Morrow configuration. We can blow down the chains starting at $(\widetilde{1,0})_{i}, i=0, \ldots, r-1$, to get a chain. To check that this chain is a Morrow configuration we must compute its determinant, which we can do with continued fractions as in [7]. We first replace the two end chains by vertices with the rational weights $-A+1-\frac{P}{Q}$ and $-\frac{q}{p}$ determined by their continued fractions to get a chain of four vertices with weights

$$
-A+1-\frac{P}{Q}, \quad 0, \quad-1+A, \quad-\frac{q}{p} .
$$

Then, computing the continued fraction for this chain based at its right vertex gives $\frac{q}{p}-\frac{Q}{P}=\frac{P q-p Q}{P p}=\frac{1}{P p}$, showing that the determinant is -1 as desired, and completing the proof.

Theorem 4.1. Given positive integers $P, Q, p, q$ with $P q-p Q=1$ and positive integers $a_{1}, \ldots, a_{r-1}$, the splice diagram of our rational polynomial $f$ of simple type with non-isotrivial fibres is given in Figure 8 with

$$
\begin{aligned}
& A=a_{1}+\cdots+a_{r-1}, \\
& B=A Q+P-Q, \\
& C=A q+p-q, \\
& b_{i}=q Q a_{i}+1 \quad \text { for each } i .
\end{aligned}
$$

The degree of $f$ is: $\operatorname{deg}(f)=A(Q+q)+P+p$.

(In [17] an "additional" case was given, which is, however, of the above type with $P=Q=p=1, q=a_{1}=2$.) 


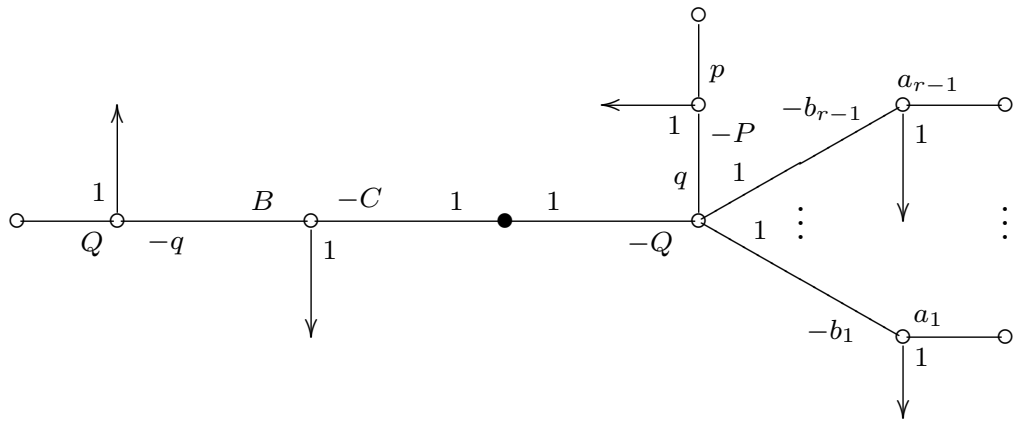

Figure 8. Splice diagram for non-isotrivial rational polynomial.

Proof. For the following computations we continue to assume the reader is familiar with the relationship between resolution graphs and splice diagrams described in [16]. The arrows signify places at infinity of the generic fibre, one on each horizontal curve. The fact that $(\widetilde{1,0})_{r}$ is next to $\widetilde{L}_{\infty}$ in the dual graph says that the edge determinant of the intervening edge is 1 . This corresponds to the fact that $P q-p Q=1$, which we already know. Similarly, $(\widetilde{1,0})_{i}$ is next to $\widetilde{L}_{\infty}$ for $i=1, \ldots, r-1$ so the weight $b_{i}$ is determined by the edge determinant condition $b_{i}=q Q a_{i}+1$. The "total linking number" at the vertex corresponding to each horizontal curve (before blowing down $\left.(\widetilde{1,0})_{0}\right)$ is zero (terminology of $\left.[\mathbf{1 6}]\right)$; this reflects the fact that the link component corresponding to the horizontal curve has zero linking number with the entire link at infinity, since at almost all points on a horizontal curve, the polynomial has no pole. The weight $C$ is determined by the zero total linking number of $(\widetilde{1,1})$, giving $C=A q+p-q$. For any $i$ the fact that vertex $(\widetilde{1,0})_{i}$ has zero total linking gives $B=A Q+P-Q$.

It is worth summarising some consequences of our construction that will be useful later.

Lemma 4.6. The number of blow-ups in the final non-separating blow-up sequence is $k:=\max \left(\left\lfloor\frac{Q}{q}\right\rfloor,\left\lfloor\frac{p}{P}\right\rfloor\right)$ and these blow-ups occurred at the $(Q,-q)$ branch or the $(p,-P)$ branch of the above splice diagram according as the first or second entry of this max is the larger. Moreover, the non-separating blow-ups occurred on the corresponding horizontal curve if and only if $q=1$ resp. $P=1$.

Proof. The first part was part of the proof of Lemma 4.5. For the second part, note that if $q=1$ then certainly $q>Q$ must fail, so $P>p$ and the non-separating blow-ups were on the left. The continued fraction on the left was $A-1+\frac{p}{q}=A-1+p$ which is integral, showing that the left chain consisted only of the exceptional curve before the non-separating blow-up. 
Conversely, if the non-separating blow-ups were adjacent to that exceptional curve then the left chain was a single vertex, hence had integral continued fraction, so $q=1$. The argument for $P=1$ is the same.

Theorem 4.2. The moduli space of polynomials $f: \mathbb{C}^{2} \rightarrow \mathbb{C}$ with the above regular splice diagram, modulo left-right equivalence (that is, the action of polynomial automorphisms of both domain $\mathbb{C}^{2}$ and range $\left.\mathbb{C}\right)$, has dimension $r+k-2$ with $k$ determined in the previous Lemma. In fact it is a $\mathbb{C}^{k}$-fibration over the $(r-2)$-dimensional configuration space of $r-1$ distinct points in $\mathbb{C}^{*}$ labelled $a_{1}, \ldots, a_{r-1}$, modulo permutations that preserve the labelling and transformations of the form $z \mapsto a z$.

Proof. The splice diagram prescribes the number of horizontal curves and the separating blow-up sequences at each point of intersection. The only freedom is in the placement of the horizontal curves in $\mathbb{P}^{1} \times \mathbb{P}^{1}$, and in the choice of points, on prescribed curves, on which to perform the string of blow-ups we call the non-separating blow-up sequence. The $(1,1)$ horizontal curve is a priori the graph of a linear map $y=a x+b$ but can be positioned as the graph of $y=x$ by by an automorphisms of the image $\mathbb{C}$.

The point in the configuration space of the Theorem determines the placement of the horizontal curves $(1,0)_{1}, \ldots,(1,0)_{r}$ (after putting the $(1,0)_{0}$ curve at $\mathbb{P}^{1} \times\{\infty\}$ and the $(1,0)_{r}$ curve at $\left.\mathbb{P}^{1} \times\{0\}\right)$. The fibre $\mathbb{C}^{k}$ determines the sequence of points for the non-separating blow-up sequence.

This proves the Theorem, except that we need to be careful, since some diagrams occur in the form of Theorem 4.1 in two different ways, which might seem to lead to disconnected moduli space. But the only cases that appear twice have four horizontal curves and the configurations $\mathcal{C}$ are related by Cremona transformations.

This completes the classification of non-isotrivial rational polynomials of simple type.

4.1. The irregular fibres. We can read off the topology of the irregular fibres of the polynomial $f$ of Theorem 4.1 from our construction, since any such fibre is the proper transform of a vertical $(0,1)$ curve together with any exceptional curves left behind as cutting divisors when blowing up on this vertical curve.

We shall use the notation $\mathbb{C}(r)$ to mean $\mathbb{C}$ with $r$ punctures (so $\mathbb{C}^{*}=$ $\mathbb{C}(1)$ ), and for the purpose of this subsection we used $C \cup C^{\prime}$ to mean disjoint union of curves $C$ and $C^{\prime}$, and $C+C^{\prime}$ to mean union with a single normal crossing. The generic fibre of $f$ is $\mathbb{C}(r+1)$.

The irregular fibres of $f$ arise through the breaking of cycles between the $(1,1)$ curve and the $(1,0)_{i}$ curve for $i=1, \ldots, r$, so there are $r$ of them. The non-separating blow-up also contributes, but it usually contributes to the $r$-th irregular fibre. However, if $P=1$ or $q=1$ then the non-separating 
blow-up occurs on a horizontal curve and can thus have any $f$-value, so it generically leads to an additional $(r+1)$-st irregular fibre.

The irregular fibres are all reduced except for the $r$-th irregular fibre, which is always non-reduced unless one of $P, Q, p, q$ is 1 .

We first assume $q \neq 1$ and $P \neq 1$, so there are exactly $r$ irregular fibres. Then for each $i=1, \ldots, r-1$ the $i$-th irregular fibre is $\mathbb{C}(r-1)+\mathbb{C}^{*}$ if $a_{i}=1$ and $\mathbb{C}(r) \cup \mathbb{C}^{*}$ if $a_{i}>1$. The $r$-th irregular fibre is $\mathbb{C}(r) \cup \mathbb{C}^{*} \cup \mathbb{C}$ generically. As mentioned above, this fibre is reduced if and only if $Q=1$ or $p=1$. There is a single parameter value in the $\mathbb{C}^{k}$ factor of the parameter space of Theorem 4.2 for which the $r$-th irregular fibre has different topology, namely $\mathbb{C}(r) \cup(\mathbb{C}+\mathbb{C})$. In this case it is non-reduced even if $Q=1$ or $p=1$.

If $q=1$ or $P=1$ then write $\frac{P}{Q}$ and $\frac{q}{p}$ as $\frac{1}{a}$ and $\frac{a k+1}{k}$ in some order. The non-separating blow-up creates irregularity in a fibre which generically is distinct from the the first $r$ irregular fibres. The generic situation is that the $r$-th irregular fibre is $\mathbb{C}(r) \cup \mathbb{C}^{*}$ or $\mathbb{C}(r-1)+\mathbb{C}^{*}$ according as $a>1$ or $a=1$ and the $(r+1)$-st irregular fibre is $\mathbb{C}(r+1) \cup \mathbb{C}$ or $\mathbb{C}(r)+\mathbb{C}$ according as $k>1$ or $k=1$, and both are reduced. But there are codimension 1 subspaces of the parameter space for which the topology is different. For instance, the $(r+1)$-st irregular fibre will be non-reduced if one blows up more than once on a vertical curve while doing the non-separating blow-up sequence that creates it.

4.2. Monodromy. We can also read off the monodromy for our polynomial $f$. Consider a generic vertical $(0,1)$ curve $C$ in our construction. Removing its intersections with the horizontal curves gives a regular fibre $F$ of $f$. Since we have positioned the horizontal curve $(1,0)_{0}$ at $\infty$ we think of $F$ as an $(r+1)$-punctured $\mathbb{C}$. We call the intersection of the $(1,1)$ horizontal curve with $C$ the 0 -th puncture of $F$ and for $i=1, \ldots, r$ we call the intersection of the $(1,0)_{i}$ curve with $C$ the $i$-th puncture of $F$.

If the $(r+1)$-st irregular fibre exists the local monodromy around it is trivial. For $i=1, \ldots, r$ the monodromy around the $i$-th irregular fibre rotates the 0 -th puncture of the regular fibre $\mathbb{C}(r+1)$ around the $i$-th puncture. In terms of the braid group on the $r+1$ punctures, with standard generators $\sigma_{i}$ exchanging the $(i-1)$-st and $i$-th puncture for $i=1, \ldots, r$, the local monodromies are $h_{1}=\sigma_{1}^{2}, h_{2}=\sigma_{1} \sigma_{2}^{2} \sigma_{1}^{-1}, \ldots, h_{r}=\sigma_{1} \ldots \sigma_{r-1} \sigma_{r}^{2} \sigma_{r-1}^{-1} \ldots \sigma_{1}^{-1}$. The monodromy $h_{\infty}=h_{r} \ldots h_{1}$ at infinity is $\sigma_{1} \sigma_{2} \ldots \sigma_{r} \sigma_{r} \ldots \sigma_{1}$. It is not hard to verify that $h_{1}, \ldots, h_{r}$ freely generate a free subgroup of the braid group.

\section{Explicit polynomials.}

The splice diagram gives sufficient information (Newton polygon, topological properties, etc.) that one can easily find the polynomial without significant 
computation by making an educated guess and then confirming that the guess is correct. The answer is as follows:

Case 1. $k \leq \frac{p}{P}<k+1$. (Then $\frac{p}{P}<\frac{q}{Q} \leq k+1$.)

Let $s_{1}=\alpha_{0}+\alpha_{1} x+\cdots+\alpha_{k-1} x^{k-1}+x^{k} y$. Let $\beta_{1}, \ldots \beta_{r-1}$ be distinct complex numbers in $\mathbb{C}^{*}$.

$$
f(x, y)=x^{q-Q k} s_{1}^{Q}+x^{p-P k} s_{1}^{P} \prod_{i=1}^{r-1}\left(\beta_{i}-x^{q-Q k} s_{1}^{Q}\right)^{a_{i}} .
$$

Case 2. $k \leq \frac{Q}{q}<k+1$. (Then $\frac{Q}{q}<\frac{P}{p} \leq k+1$.)

Let $s_{2}=\alpha_{0}+\alpha_{1} y+\cdots+\alpha_{k-1} y^{k-1}+x y^{k}$. Let $\beta_{1}, \ldots \beta_{r-1}$ be distinct complex numbers in $\mathbb{C}^{*}$.

$$
f(x, y)=y^{Q-q k} s_{2}^{q}+y^{P-p k} s_{2}^{p} \prod_{i=1}^{r-1}\left(\beta_{i}-y^{Q-q k} s_{2}^{q}\right)^{a_{i}} .
$$

One can compute the splice diagram and see it is correct. One can verify that the generic fibres are rational by the explicit isomorphism:

$$
f^{-1}(t) \rightarrow \mathbb{C}-\left\{0, \beta_{1}, \ldots, \beta_{r-1}, t\right\} \quad\left\{\begin{array}{lll}
(x, y) & \mapsto x^{q-Q k} s_{1}^{Q} \\
(x, y) & \mapsto y^{Q-q k} s_{2}^{q}
\end{array} \quad\right. \text { (Case 1), }
$$

for generic $t$. The irregular values of $t$ are $0, \beta_{1}, \ldots, \beta_{r-1}$ if $P \neq 1$ and $q \neq 1$. If $P=1$ then $t=\alpha_{0} \prod \beta_{i}$ is the additional irregular value that our earlier discussion predicts, and if $q=1$ then $t=\alpha_{0}$ is the additional irregular value.

The space of parameters $\left(\alpha_{0}, \ldots, \alpha_{k-1}, \beta_{1}, \ldots, \beta_{r-1}\right)$ maps to the moduli space we computed earlier with fibre of dimension 1 . Indeed, with $B, C$ as in Theorem 4.1, the polynomial

$$
f_{\lambda}(x, y)=\lambda^{-1} f\left(\lambda^{B} x, \lambda^{-C} y\right)
$$

has the same form with the parameters $\beta_{j}$ replaced by $\lambda^{-1} \beta_{j}$ and $\alpha_{j}$ replaced by $\lambda^{j B+A-1} \alpha_{j}$.

To put the above polynomials in the form of $f_{1}(x, y)$ of Theorem 1.1, in Case 1 we rename the exponents $q-Q k$ to $q_{1}, p-P k$ to $p_{1}, Q$ to $q, P$ to $p$. In Case 2 we rename $Q-q k$ to $q_{1}, P-p k$ to $p_{1}$, and then exchange $x$ and $y$.

\section{The isotrivial case.}

After the first version of this paper was completed we realised that the classification in [12] for the isotrivial case has omissions. In this section we therefore sketch the corrected classification using the techniques of this paper. The discussion of the parameter spaces and the irregular fibres for the resulting polynomials is similar to the non-isotrivial case, so we leave it to 
the reader. One can give an alternative proof using Kaliman's classification [9] of all isotrivial polynomials.

We will restrict ourselves to the case of ample rational polynomials, i.e., those with at least three $(1,0)$ horizontal curves. The case of one $(1,0)$ horizontal curve always gives a polynomial equivalent to a coordinate by the Abhyankar-Moh-Suzuki theorem [1, 23]. The case of two $(1,0)$ horizontal curves is dealt with from a splice diagram perspective in [15] and earlier by analytic methods in [21]. The result is included in our summary Theorem 1.1.

As before, compactify $\mathbb{C}^{2}$ to $X$ and construct a map $X \rightarrow \mathbb{P}^{1} \times \mathbb{P}^{1}$. The map is essentially canonical (up to an automorphism of one factor.) The image of the divisor at infinity $D \subset X$ in $\mathbb{P}^{1} \times \mathbb{P}^{1}$ is given by a collection of $(1,0)$ curves since we used three of the horizontal curves to get a map to $\mathbb{P}^{1} \times \mathbb{P}^{1}$ and in order that the fibres give an isotrivial family, any other horizontal curves must also be $(1,0)$ curves.

When there are at least three $(1,0)$ horizontal curves, by the following lemma the original configuration of curves in $\mathbb{P}^{1} \times \mathbb{P}^{1}$ breaks into the two cases of no vertical curves or one vertical curve.

Lemma 6.1. An ample rational polynomial with isotrivial fibres has at most one vertical curve over a finite value.

Proof. We can argue as in the previous section. The curve over infinity, $L_{\infty}$ is not blown up since there are no triple points. If there is more than one vertical curve over a finite value then there are precisely three $(1,0)$ horizontal curves since otherwise there would be at least two $(1,0)$ horizontal curves that would be blown up at least twice and since all curves beyond these horizontal curves (exceptional curves or vertical curves) have self-intersection $<-1$ we would get two branches $B_{1}$ and $B_{2}$ made up of the proper transforms of these two $(1,0)$ horizontal curves and all curves beyond these, meeting at a valency $>2$ curve, $L_{\infty}$, with self-intersection 0 . This is the impossible situation of Lemma 3.5.

There can be at most two vertical curves since if there are $l$ vertical curves we need to break $2 l$ cycles but since there are precisely three $(1,0)$ horizontal curves, we begin with $l+4$ curves so we can break at most $l+2$ cycles by Lemma 3.1 (i). Therefore $2 l \leq l+2$ so $l \leq 2$.

The lemma follows when we get rid of the case of two vertical curves and three $(1,0)$ horizontal curves. The few cases are easily dismissed by hand.

So the beginning configuration is given by Figure 9 or Figure 10 . We analyse these below as Case 1 and Case 2 .

Case 1. Denote by $r$ the number of horizontal curves. In Figure 9 we must leave behind $r-1$ curves as cutting divisors. To do so we do a non-separating 


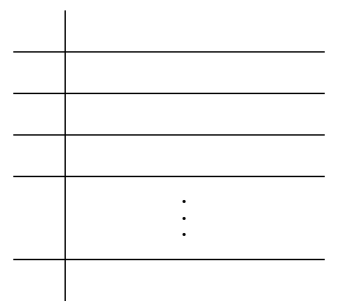

Figure 9. Configuration of horizontal curves with $L_{\infty}$.

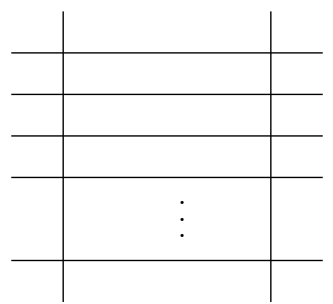

Figure 10. Configuration of horizontal curves with $L_{\infty}$ and a vertical curve over a finite value.

blow-up sequence on each of $r-1$ horizontal curves (anything else leads to a configuration of curves whose intersection matrix has determinant 0 , and which can therefore not blow down to a Morrow configuration). Thus, on the $i$-th horizontal curve we blow up $a_{i}$ times and then leave behind the final exceptional divisor, giving a string of -2 curves of length $a_{i}-1$.

The resulting splice diagram is as in Figure 11.

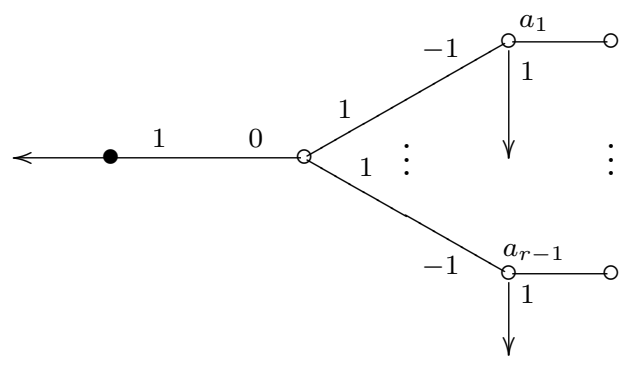

Figure 11. Splice diagram for Case 1 of isotrivial fibres. 
This splice diagram has been analysed in [16], where it is shown that its general polynomial is

$$
f(x, y)=y \prod_{i=1}^{r-1}\left(x-\beta_{i}\right)^{a_{i}}+h(x)
$$

where $h(x)$ is a polynomial of degree $<\sum_{i=1}^{r-1} a_{i}$.

This case covers the following cases from [12]: Case 1 of Theorem 3.3., Theorem 3.7, Case I of Theorem 3.10.

Case 2. Denote by $r+1$ the number of horizontal curves. In Figure 10 we must do separating blow-up sequences at $r$ intersection points and then do an additional non-separating blow-up sequence. As in Section 4, one finds that each of $r-1$ of the separating blow-up sequences creates a string of -2 curves attached to the corresponding horizontal curve, while the last one can be arbitrary, as described in the proof of Lemma 4.5. In Figure 12 we show the situation after doing the first $r-1$ separating blow-up sequences and doing the first step of the $r$-th one.

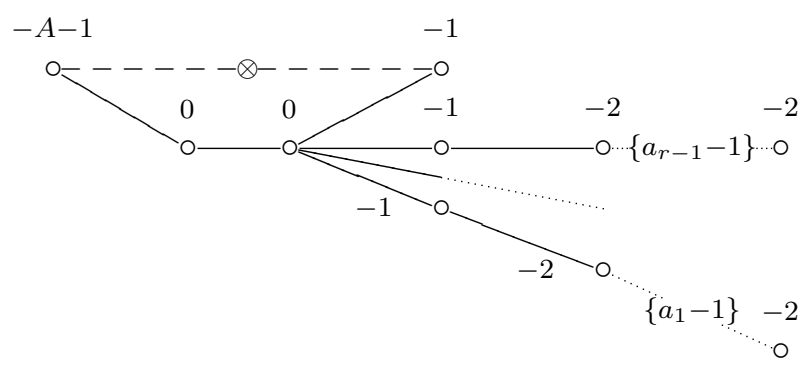

Figure 12. Dual graph of partially blown-up configuration of curves for Fig. 10.

Moreover, the non-separating blow-up sequence then occurs adjacent to the exceptional curve left behind in the final separating blow-up sequence. The analysis is almost identical to the proof of Lemma 4.5, with the resulting strings now having continued fractions $A+\frac{P}{Q}$ and $\frac{q}{p}$ respectively, with notation as in that proof.

The resulting splice diagram is as in Figure 13, with notation exactly as in Theorem 4.1. The polynomial in this case is exactly as in Section 5 except that the first term $x^{q-Q k} s_{1}^{Q}$ respectively $y^{Q-q k} s_{2}^{q}$ is omitted. Namely, let $\beta_{1}, \ldots \beta_{r-1}$ be distinct complex numbers in $\mathbb{C}^{*}$ and let $k$ be as in Theorem 4.2 . 


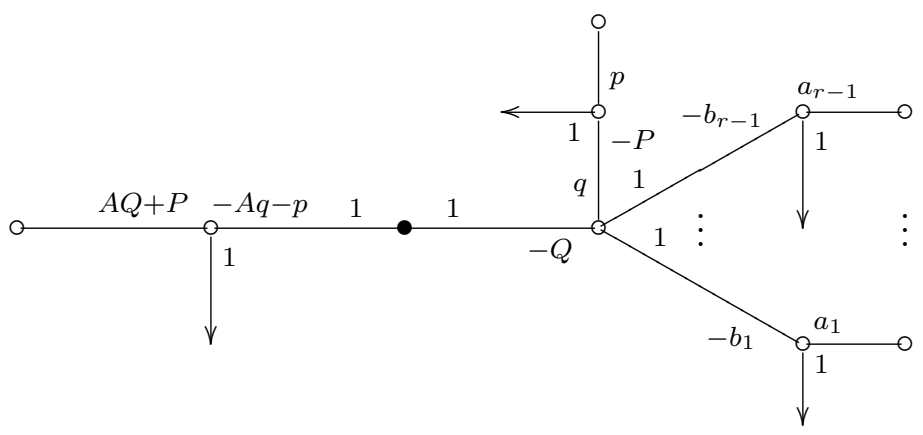

Figure 13. Splice diagram for Case 2 of isotrivial fibres.

If $k \leq \frac{p}{P}<k+1$ (so $\frac{p}{P}<\frac{q}{Q} \leq k+1$ ), let $s_{1}=\alpha_{0}+\alpha_{1} x+\cdots+\alpha_{k-1} x^{k-1}+$ $x^{k} y$. Then

$$
f(x, y)=x^{p-P k} s_{1}^{P} \prod_{i=1}^{r-1}\left(\beta_{i}-x^{q-Q k} s_{1}^{Q}\right)^{a_{i}} .
$$

If $k \leq \frac{Q}{q}<k+1$ (so $\frac{Q}{q}<\frac{P}{p} \leq k+1$ ), let $s_{2}=\alpha_{0}+\alpha_{1} y+\cdots+\alpha_{k-1} y^{k-1}+$ $x y^{k}$. Then

$$
f(x, y)=y^{P-p k} s_{2}^{p} \prod_{i=1}^{r-1}\left(\beta_{i}-y^{Q-q k} s_{2}^{q}\right)^{a_{i}} .
$$

This case covers the following cases from [12]: Cases 2, 3, 4 of Theorem 3.3 and Case II of Theorem 3.10. However, [12] only has examples in which one of $P, Q, p, q$ is equal to 1 .

Note that the isotrivial splice diagrams of Case 1 and Case 2 can be considered to belong to one family: putting $(P, Q)=(1,0)$ in Figure 13 gives Figure 11. Nevertheless, the two cases have rather different geometric properties.

\section{General rational polynomials.}

In this section we will give a result for ample rational polynomials that are not necessarily of simple type.

Proposition 7.1. An ample rational polynomial contains a $(1,0)$ horizontal curve whose proper transform has self-intersection -1 and meets $\widetilde{L}_{\infty}$.

Proof. By the classification of ample rational polynomials of simple type, the proposition is true in this case. So, we may assume that there is a horizontal curve of type $(m, n)$ for $m>1$.

Suppose there is no $(1,0)$ horizontal curve with the property of the proposition. Then by the proof of Lemma 3.4 there are at least two $(1,0)$ horizontal curves whose proper transforms have self-intersection $<-1$ and meet 
$\widetilde{L}_{\infty}$. By Lemma 3.2 any curves beyond these horizontal curves have selfintersection $<-1$.

A horizontal curve of type $(m, n)$ must meet $L_{\infty}$ at exactly one point, and hence with a tangency of order $m$ or at a singularity of the curve. This is because if a horizontal curve were to meet $L_{\infty}$ twice then we would not be able to break cycles since when we blow up next to $L_{\infty}$, those exceptional curves are sent to infinity under the polynomial and hence must be retained in the configuration of curves. Thus we must blow up there to get a configuration of curves with normal intersections. The final exceptional curve in such a sequence of blow-ups will have self-intersection -1 and valency $>2$.

If we can blow down the configuration of curves then eventually at least one curve adjacent to the -1 curve is blown down and hence the -1 curve ends up with non-negative self-intersection. But the final configuration is not a linear graph since the proper transforms of the two $(1,0)$ horizontal curves and any curves beyond give two branches. Thus the final configuration is not a Morrow configuration which contradicts Lemma 3.1.

The following result is a generalisation of Lemma 3.3.

Corollary 7.1. For any ample rational polynomial, a smooth horizontal curve of type $(m, n)$ with $m>0$ must be of type $(m, 1)$.

Proof. The statement is true for $m=1$ by Lemma 3.3 so will assume $m>1$. A curve of type $(m, n)$ will intersect the $(1,0)$ horizontal curves $m$ times, with multiplicity, unless possibly if the $(m, n)$ curve is singular at these points of intersection. The latter possibility is ruled out by the assumption of the corollary. Hence the $(1,0)$ horizontal curves will be blown up at least $m$ times and their proper transforms will have self-intersection $<-m$. This contradicts the previous proposition so the result follows.

When the rational polynomial is not ample, Russell has an example of a horizontal curve of type $(3,2)$. See the examples in the next section. Note that smoothness of the horizontal curve is necessary in the corollary (at the points of intersection with the $(1,0)$ horizontal curves) since we can always have two horizontal curves of types $(l, 1)$ and $(m, 1)$ and together they can be considered as a singular horizontal curve of type $(l+m, 2)$.

7.1. Adding horizontal curves. Consider the following construction on $\mathbb{C}^{2}$. Blow up repeatedly starting at a point on the $y$-axis so that the resulting exceptional curves form a chain from the $y$-axis to the last exceptional curve blown up. If we now remove the $y$-axis and all but the last exceptional curve from the blown-up $\mathbb{C}^{2}$ we get a new $\mathbb{C}^{2}$ that we call $\mathbb{C}_{1}^{2}$. Any polynomial $f: \mathbb{C}^{2} \rightarrow \mathbb{C}$ induces a polynomial $f_{1}: \mathbb{C}_{1}^{2} \rightarrow \mathbb{C}$. Suppose the $y$-axis intersects generic fibres of $f$ in $d$ points. Then the generic fibres of $f_{1}$ are simply generic fibres of $f$ with $d$ extra punctures. In fact, this construction simply 
adds an extra degree $d$ horizontal curve, namely the $y$-axis becomes a degree $d$ horizontal curve for $f_{1}$.

From the point of view of the polynomials, what we have done is replaced $f(x, y)$ by

$$
f_{1}(x, y)=f(x, s), \quad s=a_{0}+a_{1} x+\cdots+a_{k-1} x^{k-1}+x^{k} y,
$$

that is, we have composed $f$ with the birational morphism $(x, y) \mapsto(x, s)$ of $\mathbb{C}^{2}$.

Since one can compose $f$ first with a polynomial automorphism to raise its degree, one can easily add horizontal curves of arbitrarily high degree by this construction. This makes clear that any classification of non-simple-type polynomials must take account of this sort of operation, including composition with more complicated birational morphisms.

Although this is a complication, it can also simplify some issues.

Here is a simple illustrative example. We start with the simplest rational polynomial $g(x, y)=x$, apply a polynomial automorphism to get $f(x, y)=$ $x+y^{2}$ and then apply the above birational morphism to get $f_{1}(x, y)=$ $x+\left(a_{0}+a_{1} x+\cdots+a_{k-1} x^{k-1}+x^{k} y\right)^{2}$ with one degree one horizontal and one degree two horizontal. It is not hard to check (e.g., by listing possible splice diagrams) that this gives, up to equivalence, the only non-simple-type polynomials with generic fibre $\mathbb{C}-\{0,1\}$, so with the classification of simple type polynomials, we get:

Proposition 7.2. A polynomial with general fibre $\mathbb{C}-\{0,1\}$ is left-right equivalent to one of the form $f_{2}(x, y)$ or $f_{3}(x, y)$ of Theorem 1.1 with $r=2$ or $r=3$ respectively, or to $f(x, y)=x+\left(a_{0}+a_{1} x+\cdots+a_{k-1} x^{k-1}+x^{k} y\right)^{2}$.

This proposition also follows from Kaliman's classification [9] of isotrivial polynomials.

\section{Examples.}

It is worth including some interesting known examples of rational polynomials from the perspective used in this paper. These examples are neither of simple type nor ample.

Russell [20] (correctly presented in [3]) constructed an example of a rational polynomial with no degree one horizontal curves. This is an example of a bad field generator - a polynomial that is one coordinate in a birational transformation but not in a birational morphism. It is given by beginning with three curves in $\mathbb{P}^{1} \times \mathbb{P}^{1}$ as in Figure 14 . The $(2,1)$ curve and the $(3,2)$ curve intersect at an order three tangency and at the same point the $(3,2)$ intersects itself at a tangency. They are the two horizontal curves of the polynomial. The vertical curve is $L_{\infty}$.

The actual polynomial in this case is, with $s=x y+1$,

$$
f(x, y)=\left(y^{2} s^{4}+y(s+x y) s+1\right)\left(y s^{5}+2 x y s^{2}+x\right)
$$




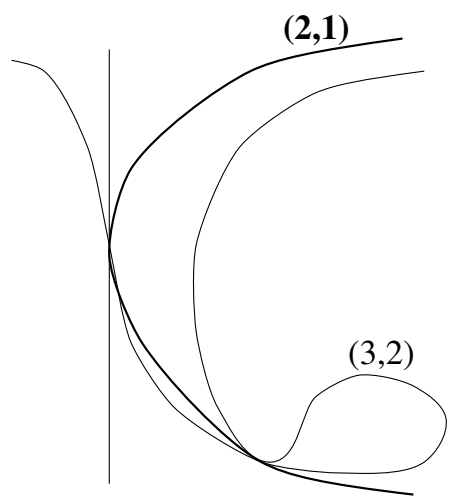

Figure 14. A bad field generator.

and the splice diagram is

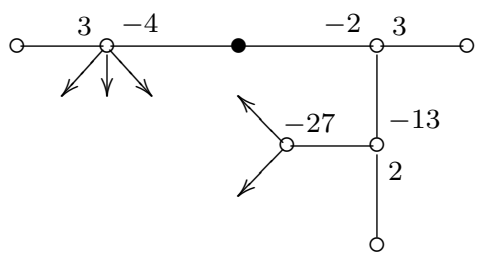

Kaliman [10] classified all rational polynomials with one fibre isomorphic to $\mathbb{C}^{*}$. Figure 15 gives three curves in $\mathbb{P}^{1} \times \mathbb{P}^{1}$, the two horizontal curves and $L_{\infty}$. The $(m, 1)$ curve has the property that when it is mapped downwards onto a $(1,0)$ curve, there are only two points of ramification, both with maximal ramification of $m$, at $L_{\infty}$ and at the irregular fibre isomorphic to $\mathbb{C}^{*}$. Kaliman's entire classification begins with this configuration of curves. The only points that can be blown up are those that are infinitely near to the point of intersection of the two horizontal curves (besides the unnecessary blowing up where the $(m, 1)$ curve meets $\left.L_{\infty}\right)$ and one exceptional curve is left behind as a component of the reducible fibre. 


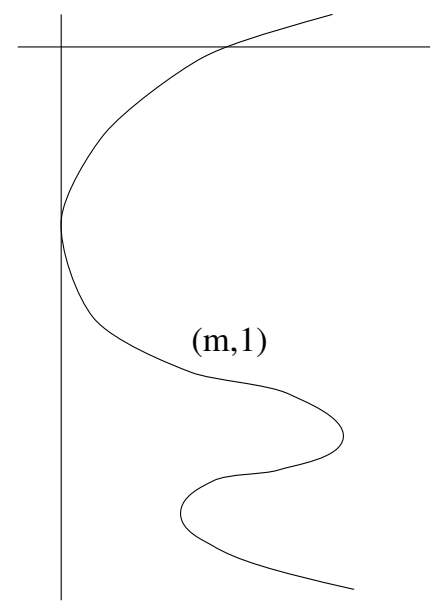

Figure 15. Classification of rational polynomials with a $\mathbb{C}^{*}$ fibre.

\section{References}

[1] S. Abhyankar and T.T. Moh, Embeddings of the line in the plane, J. Reine Angew. Math., 276 (1975), 148-166, MR 52 \#407, Zbl 0332.14004.

[2] W. Barth, C. Peters and A. van de Ven, Compact Complex Surfaces, Ergebnisse der Mathematik und ihrer Grenzgebiete (3), 4, Springer-Verlag, Berlin-New York, 1984, MR 86c:32026, Zbl 0718.14023.

[3] E. Artal Bartolo and P. Cassou-Nogues, One remark on polynomials in two variables, Pacific J. Math., 176 (1996), 297-309, MR 97m:32044, Zbl 0878.32003.

[4] E. Artal Bartolo, P. Cassou-Nogués and A. Dimca, Sur la topologie des polynômes complexes, Progress in Mathematics, 162 (1998), 317-343, MR 99k:32069, Zbl 0964.32028.

[5] D. Daigle, Birational endomorphisms of the affine plane, J. Math. Kyoto Univ., 31 (1991), 329-335, MR 92k:14012, Zbl 0766.14006.

[6] Local trees in the theory of affine plane curves J. Math. Kyoto Univ., 31 (1991), 593-634, MR 92h:14023, Zbl 0758.14018.

[7] D. Eisenbud and W.D. Neumann, Three-Dimensional Link Theory and Invariants of Plane Curve Singularities, Ann. Math. Stud., 110, Princeton, Princeton Univ. Press, 1985, MR 87g:57007, Zbl 0628.57002.

[8] P. Griffiths and J. Harris, Principles of Algebraic Geometry, John Wiley, New York, 1978, MR 80b:14001, Zbl 0408.14001.

[9] S. Kaliman, Polynomials on $\mathbb{C}^{2}$ with isomorphic generic fibres, Dokl. Akad. Nauk SSSR, 288 (1986), 39-42; English tranls. in Soviet Math. Dokl., 33 (1986), MR 87m:32009, Zbl 0623.14007.

[10] _ Rational polynomials with a $\mathbb{C}^{*}$-fiber, Pacific J. Math., 17(4) (1996), 141-194, MR 97h:14026, Zbl 0868.32010.

[11] _ Two remarks on polynomials in two variables, Pacific J. Math., 154 (1992), 285-295, MR 93b:32041, Zbl 0723.32001. 
[12] M. Miyanishi and T. Sugie, Generically rational polynomials, Osaka J. Math., 17 (1980), 339-362, MR 82e:14019, Zbl 0457.13006.

[13] J.A. Morrow, Minimal normal compactifications of $C^{2}$, in "Complex analysis, 1972 (Proc. Conf., Rice Univ., Houston, Tex., 1972), Vol. I: Geometry of singularities," Rice Univ. Studies, 59 (1973), 97-112, MR 48 \#11580, Zbl 0277.32019.

[14] W.D. Neumann, A calculus for plumbing applied to the topology of complex surface singularities and degenerating complex curves, Trans. AMS, 268 (1981), 299-344, MR 84a:32015, Zbl 0546.57002.

[15] _ Complex algebraic curves via their links at infinity, Invent. Math., 3 (1989), 445-489, MR 91c:57014, Zbl 0734.57011.

[16] _ Irregular links at infinity of complex affine plane curves, Quarterly J. Math., 50 (1999), 301-320, MR 2000i:32047, Zbl 0958.32030.

[17] W.D. Neumann and P. Norbury, Monodromy and vanishing cycles of complex polynomials, Duke Math. J., 101 (2000), 487-497, MR 2001j:32028.

[18] _ Unfolding polynomial maps at infinity, Math. Ann., 318(1) (2000), 149-180, CMP 1785580.

[19] C.P. Ramanujam, A topological characterization of the affine plane as an algebraic variety, Ann. Math., 94 (1971), 69-88, MR 44 \#4010, Zbl 0218.14021.

[20] P. Russell, Good and bad field generators, J. Math. Kyoto Univ., 17 (1977), 319-331, MR 56 \#2977, Zbl 0367.12013.

[21] H. Saito, Fonctions entière qui se reduisent à certains polynômes, I, Osaka J. Math., 9 (1972), 293-332, MR 49 \#3187, Zbl 0248.32005.

[22] _ Fonctions entière qui se reduisent à certains polynômes, II, Osaka J. Math., 12 (1977), 649-674, MR 57 \#679, Zbl 0372.32004.

[23] M. Suzuki, Propriétés topologiques des polynômes de deux variables complexes et automorphismes algébriques de l'espace $C^{2}$, J. Math. Soc. Japan, 26 (1974), 241-257, MR 49 \#3188, Zbl 0276.14001.

Received September 18, 2000 and revised February 21, 2001. This research was supported by the Australian Research Council.

Department of Mathematics

Barnard College, Columbia University

NEW YORK, NY 10027

E-mail address: neumann@math.columbia.edu

Department of Mathematics and Statistics

University of MELBOURNe

Australia 3010

E-mail address: norbs@ms.unimelb.edu.au 